\title{
X-BAND TRAVELLING WAVE ACCELERATOR STRUCTURE R\&D
}

\author{
K.Jin ${ }^{*}$, Y.J.Pei, D.M.Jiang, Y.Z.Liu, NSRL USTC, Hefei 230029, China \\ Q.J.Lai, M.H.Zhao, H.Zhao, Dept.of Phys, Nanjing Uni. Nanjing 210093, China
}

\section{Abstract}

A $1.6 \mathrm{MeV} \mathrm{X}$-Band traveling wave linear accelerator is being developed in our Lab. in view of X-band Linac $R \& D$ and industrial purposes. One section of accelerator structure has been fabricated successfully. In this paper, the RF coupler computer simulation, the constant powerloss collinear load study and the accelerator section development are described in detail. The manufacture technology and some measurement results are presented.

\section{INTRODUCTION}

The linear accelerators at an X-band frequency are being interested since it gives a reduced accelerator length and reduced average power consumption compared with lower frequencies [1]. A $1.6 \mathrm{MeV}$ traveling wave linear accelerator is being developed in our Lab. with the view of X-band R\&D. Considering the industrial purposes a magnetron with a peak power of $0.3 \mathrm{Mw}$, the frequency of $9.370 \mathrm{GHz}$ is adopted. A key part-the accelerator structure has been fabricated successfully. It consists of a RF input coupler, 38 disk-loaded accelerating cavities and a constant power-loss collinear load as shown in Fig.1. The coupler numerical simulation design, the collinear load study and the accelerator section development are described in detail as following. The manufacture technology and some measurement results are presented.

\section{RF INPUT COUPLER DESIGN}

The RF coupler is an important component for an accelerator structure. It consists of a tapered rectangular waveguide and a coupling cavity with a coupling aperture. It must be well matched to the feeding waveguide and has synchronous frequency for the proper phase advance in the structure so that ensure the input power is fed effectively and an excellent travelling wave is transmitted in the accelerating cavities train. Therefore the sizes of the coupling cavity and the coupling iris aperture should be designed exactly at operation frequency so that the optimum transmission parameter $\mathrm{S}_{21}$ and scattering parameter $S_{11}$ are obtained.

In our project, the numerical simulation design of RF coupler assembly was considered [2]. In the wake of the computer program development, special the MAFIA-code [3], it made the three-dimensions (3D) S-parameters calculation in time domain becoming possible and the change in dimensions can be easily implemented on the computer. In numerical simulation, a computer model that approximates closely real coupler cavity was built first. It consists of an input coupler, a regular accelerating cavity and an output coupler. Considering the symmetrical, only a half of the structure is needed in calculation. It is necessary to set an appropriate computation period so that assure the electric-magnetic wave can transmitted to end of output coupler (for calculation $\mathrm{S}_{21}$ ), reflection wave can return to the front end of input coupler (for calculation $\mathrm{S}_{11}$ ) and the travelling wave in the structure reach to a steady-state. Two main factors of effecting matching and tuning of coupler are coupling cavity diameter and coupling iris aperture. The S-parameters are calculated after each size changed, and the thickness of coupling iris can be obtained by edit a calculation formula in program. Revising the sizes step by step in such a way, in conjunction with microwave measurement by Kyhl method [4], the optimal matching and tuning results are acquired finally. The coupler assembly and time history of $S_{21}$ are showed in Fig.2 and Fig.3. A good result of voltage standing wave ratio measurement is achieved (see Fig.7).

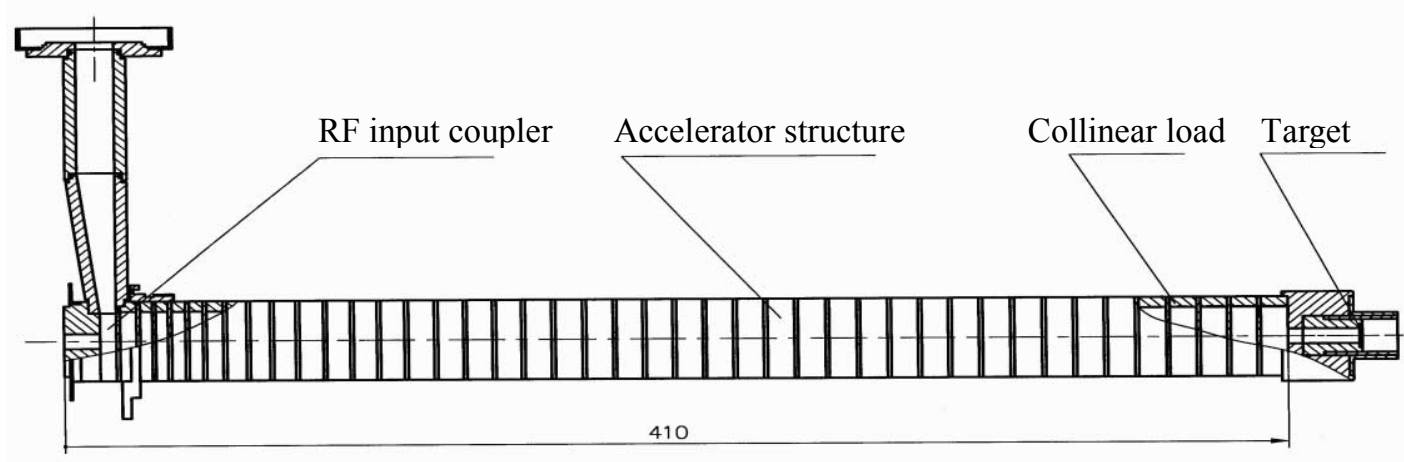

Figure 1: Sketch of 1.6MeV X-band accelerator section.

*jinkai@ustc.edu.cn 


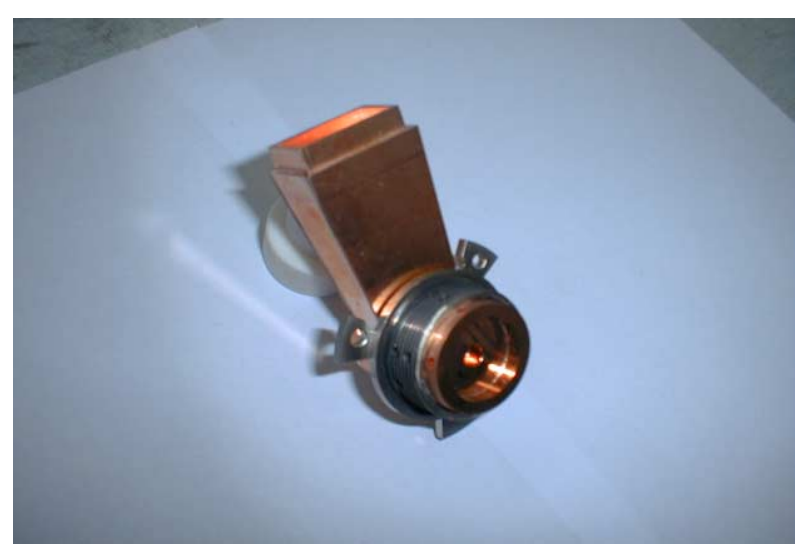

Figure 2: RF input coupler assembly.

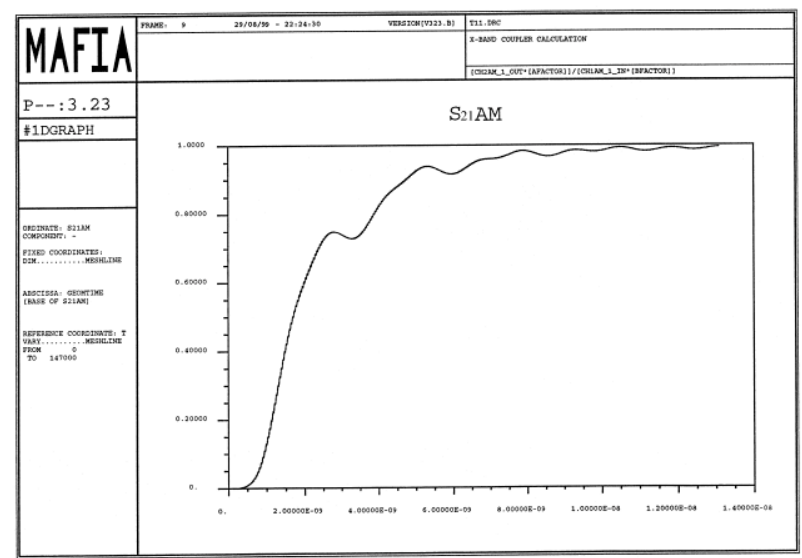

Figure 3: Amplitude of transmission wave vs time.

\section{COLLINEAR LOAD STUDY}

The typical operation of a travelling wave electron linear accelerator results in remnant RF power appearing at the termination of the accelerating structure. In general this power is extracted by an output coupler and absorbed in an external matched load in order to prevent undesirable reflection. Considering the special characteristics of the cavities coated high-resistance material on the inside walls, a technique, called collinear load, was finally adopted. It drastically increases the skin losses within the terminating cavities of the accelerator section to absorb the residual RF power and the transfer of energy between the microwave circuit and the electron beam is maintained simultaneously.

Also there are several other advantages in the collinear load as compared with a standard output coupler [5]. The collinear load is perfectly symmetric which avoids transverse excitation of the beam and intrinsically does not contribute to any emittance growth. All the higher order modes with field in the last few cells are automatically damped via dissipation in the cavity walls and it makes the accelerating structure more compact.
In our project, the constant power-loss collinear load terminating the accelerator section was developed successfully [6]. In order to ensure enough attenuation and compact structure of the load, the scheme of a fourcells structure was chosen. The "iron-chromiumaluminium alloy", a kind of high-resistance material, meets attenuation and vacuum properties quite well. The resistive films with thickness of about $50 \mu \mathrm{m}$ was coated on the inside walls of the oxygen free high conductivity copper cavities (see Fig.4.) by flame-spraying the "ironchromium-aluminium alloy" wire. The photograph of the components is shown as Fig.5. Furthermore, the combination assembly of cavities was brazed in a hydrogen furnace. Considering the constant power-loss condition, the factor of merit $\mathrm{Q}_{i}$ of each cell in the load was calculated and the relevant areas $S_{i}$ of absorbing material was coated in load. Therefore a one-way attenuation in the collinear load is: $\mathrm{A} \approx 16 \mathrm{~dB}$. And the energy gained by an electron accelerated at the crest of the travelling wave in the constant power-loss collinear load is $70 \%$ as compared to the normal constant Q-value accelerating structure. The attenuation and voltage standing wave ratio are measured. A one-way attenuation in the load is nearly $17 \mathrm{~dB}$. The voltage standing wave ratio of the turned load connecting with a matched RF input coupler, VSWR $=1.03$, was achieved at the operation frequency.

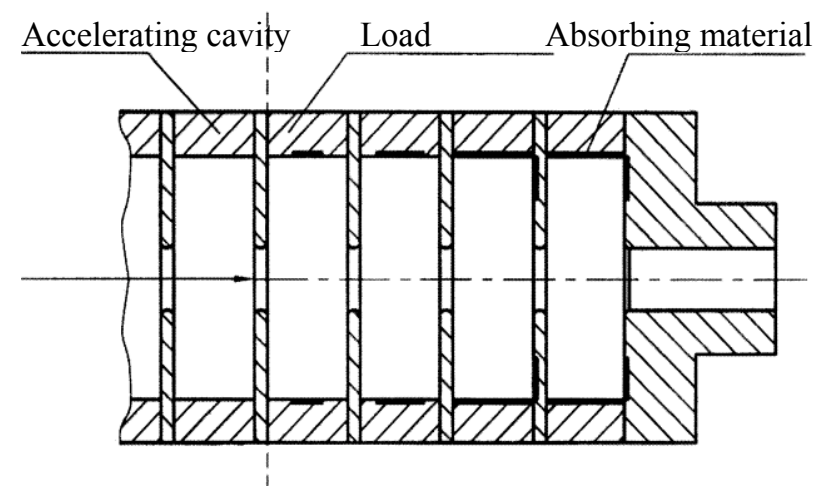

F 4: The sketch of the collinear load.

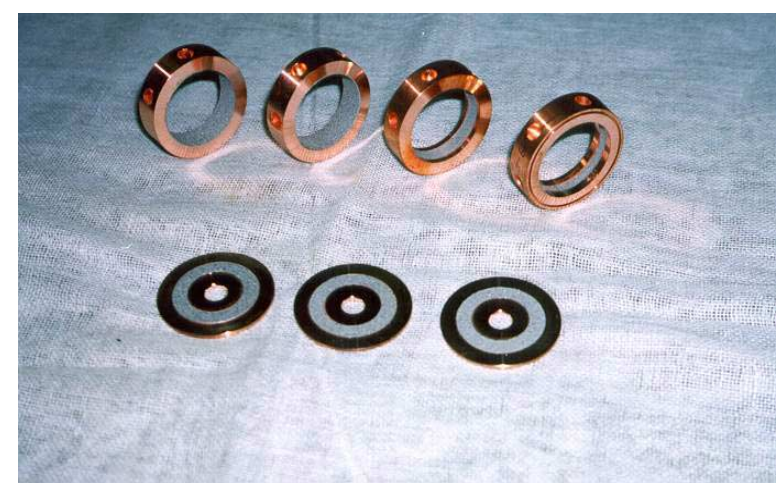

Figure 5: The components of the load with absorbing material. 


\section{ACCELERATOR SECTION DEVELOMENT}

In view of $\mathrm{X}$-band Linac R\&D and industrial purposes, a traveling wave linear accelerator with the energy of $1.6 \mathrm{MeV}$, the beam current of $65 \mathrm{~mA}$ is being developed. The magnetron with a peak power of $0.3 \mathrm{Mw}$, the frequency of $9.370 \mathrm{GHz}$ is adopted. One section of accelerator structure has been fabricated successfully. It consists of 38 disk-loaded accelerating cavities. The phase velocity of $\beta_{\mathrm{p}}$ from 0.5 to 0.97 , the operation mode of $2 \pi / 3$ and the attenuation constant of $0.48 \mathrm{Nps}$ are considered. The cavities were machined high-precisely in a constant temperature room. It was brazed with the input coupler and the collinear load in a hydrogen furnace as shown in Fig.6. Then each cell of the accelerating structure was tuned to the phase shift $\phi=120^{\circ} / \mathrm{ce} 11$. The RF parameters are measured with the Network Analyzer HP 8722D. The voltage standing wave ratio, $\mathrm{VSWR}=1.023$, was achieved at the operation frequency and VSWR is less than 1.2 in a frequency-bandwidth of $26 \mathrm{MHz}$. The measurement result of VSWR vs frequency for the typical structure is shown in Fig.7. After that the structure was welded together with an electron gun, a waveguide window and a gold target and was processed by high temperature of $500^{\circ} \mathrm{C}, 10$ hours in a vacuum outgas oven informing a compact RF vacuum assembly. Finally, an external water jacket is used so that the structure can be cooled and keep constant operating temperature. The high-power test of the accelerator section will be progressed.

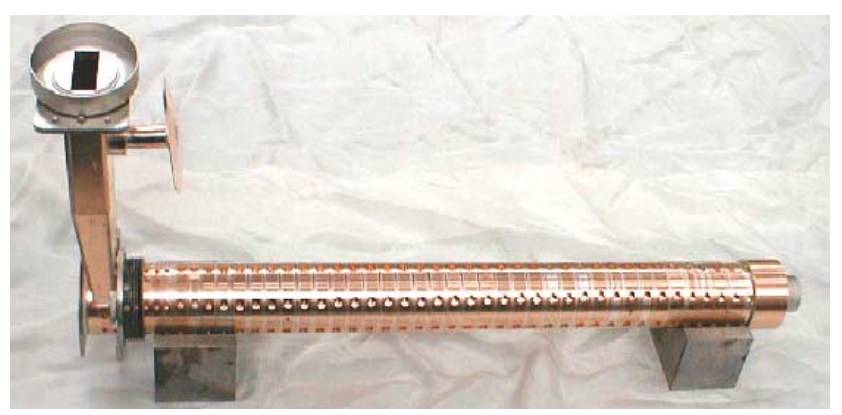

Figure 6: 1.6MeV X-band accelerator section.

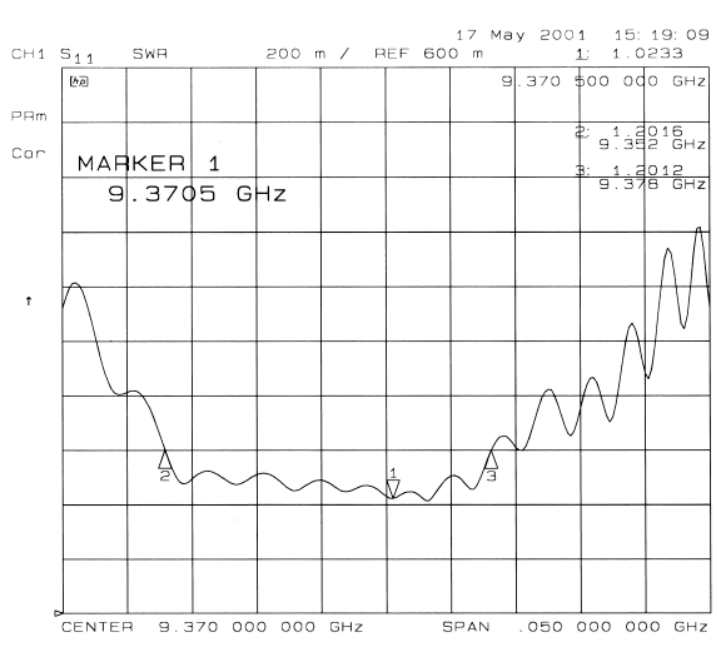

Frequency $/ \mathrm{GHz}$

Figure 7: The measurement results of VSWR vs frequency.

\section{REFERENCES}

[1] R.D. Ruth, “Linear Colliders," SLAC-PUB-5597, July 1991

[2] K. Jin, et al., "X-Band RF Coupler Numerical Simulation Design," to be contributed to APAC' 01, Sep. 2001

[3] MAFIA, the MAFIA Collaboration, December 1996.

[4] E. Westbrook, 1963, Microwave Impedance Matching of Feed Waveguide to the Disk-loaded Accelerator Structure Operating in the $2 \pi / 3$ mode, TN-63-103, SLAC, Stanford, California.

[5] Conceptual Design of a $500 \mathrm{GeV}$ e+e- Linear Collider with Integrated X-ray Laser Facility, DESY 1997-048, Volume II , p.621.

[6] K. Jin, et al., "Collinear Load Study for X-Band Linear Accelerator Structure," to be published on NIMA, 2001 